\title{
Tentative Assignments of Fundamental Vibrations of Thio- and Selenoamides. V. Tetramethylthiouram Disulfide
}

\author{
U. ANTHONI, ${ }^{a}$ G. BORCH, ${ }^{b}$ P. KLÆBOE ${ }^{c}$ and P. H. NIELSEN ${ }^{a}$
}

\begin{abstract}
${ }^{a}$ Chemical Laboratory II, The H. C. Ørsted Institute, DK-2100 Copenhagen, Denmark, ${ }^{\mathrm{b}}$ Chemistry Department A, The Technical University of Denmark, DK-2800 Lyngby, Denmark and ${ }^{\mathrm{c}}$ Department of Chemistry, University of Oslo, Oslo 3, Norway
\end{abstract}

The infrared and Raman spectra of tetramethylthiouram disulfide (TMTD) and four ${ }^{13} \mathrm{C},{ }^{15} \mathrm{~N}$ and deuterium labelled analogues have been studied in the solid state and in solution in the region below $4000 \mathrm{~cm}^{-1}$. A normal coordinate analysis has been accomplished for these compounds as a 24-body problem utilizing a general valence force field. By fitting 26 force constants to reproduce $\mathrm{ca}$. 300 observed frequencies, the normal modes of vibration are described in terms of the potential energy distribution between the symmetry coordinates. A tentative assignment of the fundamentals of TMTD and the perdeuterated compound is given based upon intensities, Raman depolarisation ratios, the ${ }^{13} \mathrm{C} /{ }^{15} \mathrm{~N}$ shifts and the calculated values. The results indicate the need for revision of previous assignments for TMTD.

In the present paper, the IR and Raman spectra of tetramethylthiouram disulfide, $\left[\left(\mathrm{CH}_{3}\right)_{2} \mathrm{NCSS}\right]_{2}$ (TMTD), and the four isotopically substituted derivatives $\quad\left[\left(\mathrm{CD}_{3}\right)_{2} \mathrm{~N}-\mathrm{CSS}\right]_{2} \quad\left(\mathrm{TMTD}-d_{12}\right)$, $\left[\left(\mathrm{CH}_{3}\right)_{2} \mathrm{~N}-{ }^{13} \mathrm{CSS}\right]_{2}\left(\mathrm{TMTD}-{ }^{13} \mathrm{C}\right),\left[\left(\mathrm{CH}_{3}\right)_{2}{ }^{15} \mathrm{~N}-\right.$ $\mathrm{CSS}]_{2}\left(\mathrm{TMTD}-{ }^{15} \mathrm{~N}\right)$, and $\left[\left(\mathrm{CD}_{3}\right)_{2} \mathrm{~N}-{ }^{13} \mathrm{CSS}\right]_{2}$ (TMTD- $d_{12}-{ }^{13} \mathrm{C}$ ) were measured in solution and in the solid state in the region below $4000 \mathrm{~cm}^{-1}$. Semiquantitative Raman depolarisation data were also obtained, but with a few exceptions vibrations of species $a$ and $b$ overlap completely. The assignments were supported by the results of a full normal coordinate analysis (NCA) (i.e. including treatment of the methyl groups) based upon the general valence force field (GVFF) derived for related compounds, ${ }^{1,2}$ assuming $C_{2}$ symmetry.

The IR and Raman spectra of TMTD have previously been measured by Coleman et al. ${ }^{3}$ in the region below $2000 \mathrm{~cm}^{-1}$. A partial assignment of the fundamental vibrations was proposed from a simplified NCA treating the methyl groups as point masses. The isotopic shifts observed in our spectra were incompatible with the assignment proposed by Coleman et al. and it has been necessary to reinvestigate the spectrum of TMTD in some detail. A reproduction of the IR spectrum of TMTD in the range $4000-250 \mathrm{~cm}^{-1}$ has been reported ${ }^{4}$ for use as reference spectrum in pesticide research. Contreras and Cortés ${ }^{5}$ reported some major IR shifts of TMTD on complex formation with cobalt(II).

\section{STRUCTURE}

In a preliminary report ${ }^{6}$ the crystal and molecular structure of TMTD has been given but has since been refined by least-squares methods to $R=0.095$ for 426 non-zero observed reflections (personal communication from the author). TMTD consists of two planar $\mathrm{C}_{2} \mathrm{NCS}_{2}$ units with a dihedral $\mathrm{C}-\mathrm{S}-\mathrm{S}-\mathrm{C}$ angle of $88.3^{\circ}$. The most important bond distances are: $\mathrm{CH}_{3}-\mathrm{N}: 1.483(21)$ and $1.506(18) \AA, \mathrm{C}-\mathrm{N}: 1.319(15) \AA, \mathrm{C}=\mathrm{S}: 1.634(13) \AA$, $\mathrm{C}-\mathrm{S}: 1.829(15) \AA$, and $\mathrm{S}-\mathrm{S}: 2.005(7) \AA$. The positions of the hydrogen atoms were estimated by assuming tetrahedral configuration of the methyl group and standard $\mathrm{C}-\mathrm{H}$ distances. The final Cartesian coordinates correspond to an overall $C_{2}$ symmetry and may be obtained on request.

${ }^{1} \mathrm{H}$ NMR studies of TMTD in solution indicate a restricted rotation around the $\mathrm{C}(\mathrm{S})-\mathrm{N}$ bond with an energy barrier of $c a .15 \mathrm{kcal} / \mathrm{mol}^{6-10}$ Splittings due to the restricted rotation around the $\mathrm{C}-\mathrm{S}$ and 
$\mathrm{S}-\mathrm{S}$ bonds have not been observed in these spectra. Likewise the ${ }^{13} \mathrm{C}$ NMR spectrum of TMTD $\left(\mathrm{CDCl}_{3}\right)$ showed only splittings due to the $\mathrm{C}-\mathrm{N}$ barrier at temperatures down to $-15^{\circ} \mathrm{C}$, the lowest temperature obtainable without the precipitation of TMTD. Probably the barriers around the $\mathrm{C}-\mathrm{S}$ and $\mathrm{S}-\mathrm{S}$ bonds do not exceed $10 \mathrm{kcal} / \mathrm{mol} .{ }^{11} \mathrm{On}$ the NMR time scale, TMTD can therefore be considered composed by two planar dimethyldithiocarbamate units joined by the $\mathrm{S}-\mathrm{S}$ bond, i.e., the $C_{2}$-symmetry is retained. Since new bands do not appear in the vibrational spectra of TMTD on dissolution we assume TMTD to occur in solution only as one isomer most probably of $C_{2}$-symmetry.

\section{EXPERIMENTAL}

The preparation of the compounds as well as the techniques and equipment used for recording the IR and Raman spectra have been given in a previous paper. ${ }^{1}$ Infrared spectra were also run in $\mathrm{CCl}_{4}$ and $\mathrm{CS}_{2}$ solution but the spectra were identical within a few $\mathrm{cm}^{-1}$ with those recorded in $\mathrm{CHCl}_{3}$ and no indication of the presence of more than one isomer was found.

Noise-decoupled ${ }^{13} \mathrm{C}$ NMR spectra were determined with a Bruker WH 90 system (22.63 MHz) operating in the Fourier transform mode. The samples were examined as saturated solutions in $\mathrm{CDCl}_{3}$ and shieldings measured relative to internal TMS. The following values were obtained at room temperature: 42.0 and $47.5 \mathrm{ppm}\left(\mathrm{CH}_{3}\right)$ and 193.5 $\mathrm{ppm}(C S)$. The spectrum was also obtained of the ${ }^{13} \mathrm{C}$ substituted TMTD, but we were unable to discern and long-range coupling due to the ${ }^{13} \mathrm{C}-\mathrm{S}-\mathrm{S}-{ }^{13} \mathrm{C}$ grouping.

\section{NORMAL COORDINATE ANALYSIS}

The NCA was performed as a 24-body problem assuming $C_{2}$-symmetry of TMTD. Treatment of TMTD under point group $C_{2}$ reduced the normal modes of vibration to the irreducible representation $T_{\text {vib }}=34 A+32 B$, and the standard $\mathrm{GF}^{12}$ programs $^{13}$ were employed. The potential field employed was of the general valence force field type similar to that described in previous papers. ${ }^{1}$ Of the 66 normal modes of vibration possible, 32 are outof-phase combinations (species $B$ ) of the two dimethyldithiocarbamate units joined by the $\mathrm{S}-\mathrm{S}$ bond. The remaining 34 of species $A$ are composed of the 32 corresponding in-phase combinations together with the $\mathrm{S}-\mathrm{S}$ stretching and $\mathrm{C}-\mathrm{S}-\mathrm{S}-\mathrm{C}$

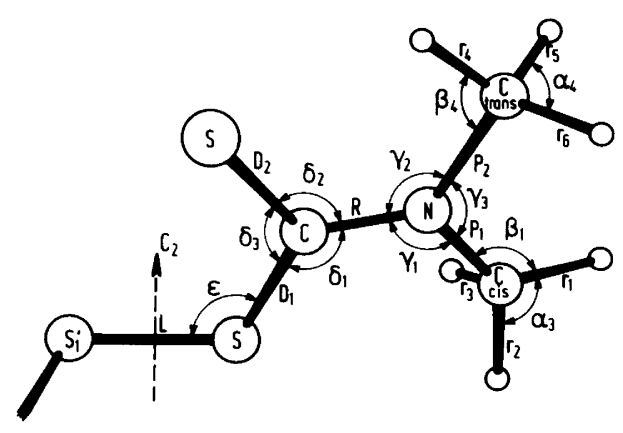

Fig. 1. Internal coordinates for TMTD (one half shown). The following coordinates are not shown on the figure: $\mathrm{CH}_{\frac{\text { cis }}{3}}-\mathrm{N}$ torsion $\tau_{1}, \mathrm{CH}_{\frac{\text { trans }}{3}}-\mathrm{N}$ torsion $\tau_{2}, \mathrm{CH}_{\frac{\mathrm{cis}}{3}}-\mathrm{N}-\mathrm{C}-\mathrm{S}$ torsion $\tau_{3}, \mathrm{CH}_{3}^{\frac{3}{\text { rans }}}-\mathrm{N}$ $-\mathrm{C}-\mathrm{S}$ torsion $\tau_{4}, \mathrm{~N}-\mathrm{C}-\mathrm{S}-\mathrm{S}$ torsion $\tau_{5}, \mathrm{~S}=$ $\mathrm{C}-\mathrm{S}-\mathrm{S}$ torsion $\tau_{6}$, and $\mathrm{S}-\mathrm{S}$ torsion $\tau_{7}$. The twofold axis of symmetry indicated is perpendicular to the plane of the paper.

torsional modes. The internal coordinates used in setting up the $F$ matrix are simply the changes in bond lengths and interbond angles. The symmetry coordinates are defined by the in-phase and out-ofphase combinations (Fig. 1).

The initial values for the force constants were first chosen in accordance with the NCA reported by Coleman et l. $^{3}$ but only a very poor agreement was achieved especially for the isotopic shifts. The values were then adjusted to comply with the force fields previously obtained for tetramethylthiourea ${ }^{1}$ and dimethyldithiocarbamates. ${ }^{2}$ This allowed assignment of more than 250 experimental frequencies by slight refinements of the diagonal elements of the $F$ matrix using the Jacobian matrix of frequencies $v s$. force constants as a guide. However, attempts to refine this force field by the usual least-squares procedure failed and led only to non-converging solutions. Inspection of the correlation matrix ( $c f$. Knoeck and Witt ${ }^{14}$ ) showed that several of the matrix elements related to the coordinates $\gamma_{1} / \gamma_{2}$ and $P_{1} / P_{2}$ were approaching 1 . Accordingly, the isotopic substitution is insufficient for determining separate force fields for the cis and trans methyl groups, and the constraints $F_{\mathrm{R}_{\gamma 1}}=F_{\mathrm{R}_{\gamma 2}}, F_{\mathrm{P}_{1} 1}=$ $F_{\mathrm{P}_{2 \gamma 2}}$ and $F_{\mathrm{P}_{1 \gamma 3}}=F_{\mathrm{P}_{2 \gamma 3}}$ were introduced. Furthermore, the number of torsional force constants was reduced by setting $H_{\tau_{3}}=H_{\tau_{4}}$ and $H_{\tau_{5}}=H_{\tau_{6}}$. A new least-square adjustment of the force field to the observed frequencies revealed that several of the important interaction force constants (e.g. $\left.F_{\mathrm{D}_{1} \mathrm{D}_{2}}\right)$ 
Table 1. Final valence force constants for tetramethylthiouram disulfide.

\begin{tabular}{|c|c|c|c|c|c|}
\hline Force type & $\begin{array}{l}\text { Constants } \\
\text { symbol }\end{array}$ & Group & $\begin{array}{l}\text { Coordinate (s) } \\
\text { Involved }\end{array}$ & $\begin{array}{l}\text { Atoms common } \\
\text { to interacting } \\
\text { coordinates }\end{array}$ & value ${ }^{b}$ \\
\hline \multirow[t]{6}{*}{ Stretch } & $\mathrm{k}_{\mathrm{r}}$ & $\mathrm{CH}_{3}$ & $C-H$ & & 4.69 \\
\hline & $k_{p}$ & $\mathrm{CH}_{3}-\mathrm{N}$ & $\mathrm{C}-\mathrm{N}$ & & 5.00 \\
\hline & $\mathrm{K}_{\mathrm{R}}$ & $N-C(S)$ & $\mathrm{N}-\mathrm{C}$ & & 6.81 \\
\hline & $\mathrm{K}_{\mathrm{D}_{2}}$ & $C=S$ & $C=S$ & & 3.02 \\
\hline & $\mathrm{K}_{\mathrm{D}_{1}}$ & $c-s$ & $c-s$ & & 2.13 \\
\hline & $k_{L}$ & $s-s$ & $s-s$ & & 2.58 \\
\hline \multirow[t]{4}{*}{ Stretch-Stretch } & $F_{r}$ & $\mathrm{CH}_{3}$ & $\mathrm{C}-\mathrm{H}, \mathrm{C}-\mathrm{H}$ & c & 0.038 \\
\hline & $\mathrm{F}_{\mathrm{RD}_{1}}$ & $N-C-s$ & $\mathrm{~N}-\mathrm{C}, \mathrm{C}-\mathrm{s}$ & c & 1.52 \\
\hline & $\mathrm{F}_{\mathrm{RP}}$ & $\mathrm{CH}_{3}-\mathrm{N}-\mathrm{C}$ & $\mathrm{C}-\mathrm{N}, \mathrm{N}-\mathrm{C}$ & $\mathbf{N}$ & 0.27 \\
\hline & $F_{p}$ & $\mathrm{CH}_{3}-\mathrm{N}-\mathrm{CH}_{3}$ & $\mathrm{C}-\mathrm{N}, \mathrm{N}-\mathrm{C}$ & N & 0.30 \\
\hline \multirow[t]{7}{*}{ Bend } & $H_{a}$ & $\mathrm{CH}_{3}$ & $<\mathrm{HCH}$ & & 0.524 \\
\hline & $\mathrm{H}_{B}$ & $\mathrm{CH}_{3}-\mathrm{N}$ & $<\mathrm{HCN}$ & & 0.773 \\
\hline & ${ }_{r_{1}}$ & $\mathrm{CH}_{3}-\mathrm{N}-\mathrm{C}$ & $<\mathrm{CNC}$ & & 1.59 \\
\hline & $\mathrm{H}_{\mathrm{r}_{3}}$ & $\mathrm{CH}_{3}-\mathrm{N}-\mathrm{CH}_{3}$ & $<\mathrm{CNC}$ & & 2.26 \\
\hline & $\mathrm{H}_{\delta_{1}}=\mathrm{H}_{\delta_{2}}$ & $\mathrm{~N}-\mathrm{C}=\mathrm{S} / \mathrm{N}-\mathrm{C}-\mathrm{S}$ & $<$ NCS & & 0.68 \\
\hline & $\mathrm{H}_{\delta_{3}}$ & $s=c-s$ & <scs & & 1.29 \\
\hline & ${ }_{E}{ }_{\varepsilon}$ & c-s-s & $<\mathrm{css}$ & & 0.83 \\
\hline \multirow[t]{4}{*}{ Stretch-bend } & $\mathrm{F}_{\mathrm{RY}}$ & $\mathrm{CH}_{3}-\mathrm{N}-\mathrm{C}$ & $<\mathrm{CNC}, \mathrm{N}-\mathrm{C}$ & $\mathrm{N}-\mathrm{C}$ & 0.68 \\
\hline & $P_{2} r_{2}$ & $\mathrm{CH}_{3}-\mathrm{N}-\mathrm{C}$ & $<\mathrm{CNC}, \mathrm{CH}_{3}-\mathrm{N}$ & $\mathrm{CH}_{3}-\mathrm{N}$ & 0.90 \\
\hline & $F_{P_{1} Y_{3}}=F_{P_{2} \gamma_{3}}$ & $\mathrm{CH}_{3}-\mathrm{N}-\mathrm{CH}_{3}$ & $<\mathrm{CNC}, \mathrm{CH}_{3}-\mathrm{N}$ & $\mathrm{CH}_{3}-\mathrm{N}$ & 1.33 \\
\hline & $F_{\mathrm{PB}}$ & $\mathrm{CH}_{3}-\mathrm{N}$ & $<\mathrm{HCN}, \mathrm{CH}_{3}-\mathrm{N}$ & $\mathrm{CH}_{3}-\mathrm{N}$ & 0.34 \\
\hline Bend-bend & $F_{B}$ & $\mathrm{CH}_{3}-\mathrm{N}$ & $<\mathrm{HCN},<\mathrm{HCN}$ & $\mathrm{C}-\mathrm{N}$ & -0.047 \\
\hline \multirow[t]{4}{*}{ Torsion } & $\mathrm{H}_{\tau_{1}}=\mathrm{H}_{\tau_{2}}$ & $\mathrm{CH}_{3}-\mathrm{N}$ & $\mathrm{CH}_{3}-\mathrm{N}$ & & 0.0314 \\
\hline & $H_{\tau_{3}}^{1}=H_{T_{4}}$ & $\mathrm{~N}-\mathrm{Cs}_{2}$ & $\mathrm{~N}-\mathrm{Cs}_{2}$ & & 0.305 \\
\hline & $H_{\tau_{5}}=H_{\tau_{6}}$ & $c-s$ & $c-s$ & & 0.177 \\
\hline & $\mathrm{H}_{\tau_{7}}$ & $s-s$ & $s-s$ & & 0.198 \\
\hline
\end{tabular}

\footnotetext{
The force constants referring to the $\mathrm{CH}_{3}^{\mathrm{Cl}}$ and $\mathrm{CH}_{3}^{\text {trans }}$ groups have been assumed identical.

${ }^{b}$ In units of mdyn/A (stretch constants), mdyn/rad (stretch-bend interaction constants) and mdyn $\cdot A /(r a d)^{2}$ (bending and torsion constants?.
}

were very poorly determined, and that a drastic reduction in the number of force constants was necessary. Using only the 26 force constants of Table 1 we finally obtained satisfactory convergence and good agreement between observed and calculated frequencies and ${ }^{15} \mathrm{~N} /{ }^{13} \mathrm{C}$ shifts (Table 2 and 3). However, the number of independent constants of the force field has been reduced to the point where detailed considerations based upon their relative values are not clearly justified. We shall only point out that the gross features are in accordance with expectations (e.g. $K_{\mathrm{D}_{2}}>K_{\mathrm{D}_{1}}$, $K_{\mathrm{R}}>K_{\mathrm{P}}$ ) and that the final values are physically reasonable.

\section{RESULTS AND DISCUSSION}

The IR spectrum of TMTD in the range 250$4000 \mathrm{~cm}^{-1}$ is reproduced in a collection of reference spectra ${ }^{4}$ and the region below $2000 \mathrm{~cm}^{-1}$, together with the Raman spectrum, in Coleman's paper. $^{3}$ The IR spectra correspond in detail to the spectra obtained by us except for a splitting in the strong band near $1500 \mathrm{~cm}^{-1}$ into two branches at 1497 and $1506 \mathrm{~cm}^{-1}$ attributed ${ }^{3}$ to the in-phase and out-of-phase $\mathrm{CN}$ stretching modes of species $a$ and $b$. This splitting does not appear in any of our spectra in the solid phase nor in solution, and the calculations indicate (Table 2) that the fundamentals in question $\left(v_{7} a\right.$ and $\left.v_{41} b\right)$ are accidentally degener-

Acta Chem. Scand. A 32 (1978) No. 8 
ate. The agreement of our Raman spectra with that given by Coleman et al. is good.

The ${ }^{13} \mathrm{C}$ and ${ }^{15} \mathrm{~N}$ shifts included in Tables 2 and 3 are not in all cases definite though the quality of the spectra is excellent. For example, the IR spectrum of TMTD displays two very strong bands at
970 and $977 \mathrm{~cm}^{-1}$ together with a weaker band at $952 \mathrm{~cm}^{-1}$. In the ${ }^{13} \mathrm{C}$-labelled compounds we observe the corresponding three bands at 974 (medium strength), 954 (strong), and $939 \mathrm{~cm}^{-1}$ (very strong). Based upon the results of the NCA this is explained (Table 2) as a shift of the funda-

Table 2. Observed and calculated vibrational frequencies $\left(\mathrm{cm}^{-1}\right)$ of tetramethylthiouram disulfide and frequency shifts obtained by comparison with $\left.\left[\mathrm{CH}_{3}\right)_{2} \mathrm{~N}-{ }^{13} \mathrm{CSS}\right]_{2}$ and $\left[\left(\mathrm{CH}_{3}\right)_{2}{ }^{15} \mathrm{~N}-\mathrm{CSS}\right]_{2}$.

\begin{tabular}{|c|c|c|c|c|c|c|c|c|c|c|c|}
\hline \multicolumn{4}{|c|}{ Infrared $^{\mathrm{a}}$} & \multicolumn{4}{|c|}{ Raman $^{\mathbf{a}}$} & \multirow{2}{*}{\multicolumn{3}{|c|}{ Calculated }} & \multirow{3}{*}{$\begin{array}{l}\text { Assignment and Description }{ }^{c} \\
\left(\mathrm{PtD}, \mathrm{Q}^{\mathrm{d}}\right)^{\mathrm{d}}\end{array}$} \\
\hline \multicolumn{3}{|c|}{ solid, disc } & \multirow{2}{*}{$\begin{array}{c}\text { solution } \\
\mathrm{CHCl}_{3}\end{array}$} & \multicolumn{3}{|c|}{ sol1d } & \multirow{2}{*}{$\begin{array}{l}\text { solution } \\
\mathrm{CHCl}_{3}\end{array}$} & & & & \\
\hline parent & ${ }^{13} \mathrm{C}-\mathrm{sh}$ & ${ }^{15} \sqrt{n-s h}$ & & parent & ${ }^{13} \mathrm{c}-\mathrm{sh}$ & ${ }^{15} \mathrm{~N}-\mathrm{sh}$ & & parent & ${ }^{13} \mathrm{c}-\mathrm{sh}$ & ${ }^{15} \mathrm{~N}-\mathrm{sh}$ & \\
\hline \multirow[t]{3}{*}{$3010 \mathrm{w}, \mathrm{br}$} & o & $\circ$ & & $3017 w$ & $\circ$ & $\circ$ & & & & & \\
\hline & & & & $2980 \mathrm{w}$ & $\circ$ & $\circ$ & $2988 \mathrm{~m}(0.41)$ & & & & \\
\hline & & & & $2960 \mathrm{w}, \mathrm{sh}$ & $\circ$ & $\circ$ & & & & & \\
\hline \multirow[t]{2}{*}{$2932 \mathrm{~m}$} & o & $\circ$ & $2938 \mathrm{~m}$ & 29338 & 0 & 0 & $2936:(0.14)$ & $\left\{\begin{array}{l}2954 \\
2949 \\
2947 \\
2947\end{array}\right.$ & $\begin{array}{l}\circ \\
: \\
\circ \\
\circ\end{array}$ & $\begin{array}{l}\circ \\
\circ \\
\circ \\
\circ\end{array}$ & 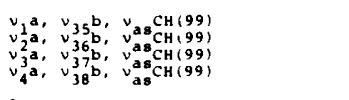 \\
\hline & & & & $2924 \mathrm{~m}, \mathrm{sh}$ & $\circ$ & o & & & & & $2 \cdot \mathrm{v}_{8} \mathrm{~A}$ \\
\hline $2895 \mathrm{vw}$ & & & & $2893 \mathrm{~m}, \mathrm{sh}$ & $\circ$ & $\circ$ & & & & & $v_{B}+v_{11} A$ \\
\hline \multirow[t]{4}{*}{$2851 \mathrm{w}$} & o & 2 & $2860 \mathrm{w}$ & $2859 \mathrm{~m}$ & 0 & $\circ$ & $2868 \mathrm{~m}(0.19)$ & $\left\{\begin{array}{l}2866 \\
2866\end{array}\right.$ & $\stackrel{\circ}{\circ}$ & $\stackrel{\circ}{\circ}$ & 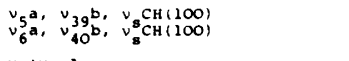 \\
\hline & & & & $2854 \mathrm{w}, \mathrm{sh}$ & & & $2857 w, 8 \mathrm{sh}$ & & & & $v_{8}+v_{12} A$ \\
\hline & & & & $2848 \mathrm{w}$ & 2 & & & & & & $v_{10}+v_{12} A$ \\
\hline & & & & $2808 w$ & -1 & $\circ$ & $2809 w(0.08)$ & & & & $2 \cdot v_{12^{A}}$ \\
\hline \multirow[t]{2}{*}{$2790 v w$} & & & $2800 \mathrm{vw}$ & $2787 w$ & & 2 & $2793 \mathrm{vw}, \mathrm{sh}$ & & & & $v_{12}+v_{13^{A}}$ \\
\hline & & & & $2508 \mathrm{vw}$ & $\circ$ & $\circ$ & $1508 \mathrm{vw}, \mathrm{sh}$ & & & & $v_{18}+v_{24} A$ \\
\hline $1500 \mathrm{~s}$ & 18 & 21 & $1504 \mathrm{~s}$ & l1490vw & & & $1498 \mathrm{~m}$, bro. 56$)$ & 1493 & 10 & 12 & $v_{7} a, v_{41} b, \underset{{ }_{P C H}(24)}{v C N(29)}{ }^{\delta C_{3}}(36)$ \\
\hline \multirow[t]{2}{*}{$1454 \mathrm{w}, \mathrm{sh}$} & o & & & $1465 \mathrm{~m}, \mathrm{br}$ & 6 & 2 & ca. $1453 \mathrm{~m}, \mathrm{br}$ & $\left\{\begin{array}{l}1466 \\
1462 \\
1459\end{array}\right.$ & $\begin{array}{l}\circ \\
\stackrel{0}{\circ} \\
\circ\end{array}$ & $\begin{array}{l}\circ \\
\circ \\
\circ\end{array}$ & 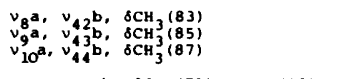 \\
\hline & & & & $1433 w$ & o & -1 & & 1442 & 7 & 7 & $v_{11} a, v_{45} b, \delta C_{3}(71), \quad \cup C N(18)$ \\
\hline $1400 \mathrm{~m}$ & 1 & 1 & $1409 w$ & $1400 \mathrm{~s}$ & 1 & $\circ$ & $1408 \mathrm{~m}(0.85)$ & 1407 & 1 & 1 & $v_{12} a, v_{46} b, 8 C_{3}(100)$ \\
\hline $1375 s$ & 8 & 9 & $1380 \mathrm{~s}$ & 13758 & 8 & 9 & $1382 \mathrm{~s}(0.67)$ & 1385 & 7 & 5 & $v_{13^{a}}, v_{4} 7^{b}, 8 \mathrm{CH}_{3}(84), v C N(17)$ \\
\hline $1335 \mathrm{vw}$ & & & $1335 \mathrm{vw}$ & $1336 \mathrm{vw}$ & -1 & & $1335 \mathrm{vw}$ & & & & $2 \cdot v_{21}{ }^{A}$ \\
\hline $1294 \mathrm{vw}$ & 16 & 1 & $1297 \mathrm{vw}$ & & & & & & & & $v_{53}+v_{59} A$ \\
\hline $1235 \mathrm{~s}$ & 4 & 19 & 12458 & $1239 w$ & 4 & 20 & ca. $1245 w$ & 1248 & 6 & 18 & $v_{14} a, v_{48} b,{ }_{6 C_{3}}^{\mathrm{CCH}_{3}}+\mathrm{NC}(33) ;{ }_{{ }_{0} \mathrm{CH}_{3}}(25)$ \\
\hline $1150 s$ & $15 / 4$ & 1 & $1152 \mathrm{~s}$ & 11508 & $13 / 0$ & $\begin{array}{l}0 \\
3\end{array}$ & $\begin{array}{l}1156(0.58) \\
1131 \mathrm{vw}, \mathrm{sh}\end{array}$ & $\left\{\begin{array}{l}1158 \\
1155\end{array}\right.$ & 12 & 2 & 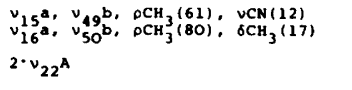 \\
\hline $1110 v w$ & 2 & 3 & $1115 \mathrm{w}, \mathrm{sh}$ & $1105 w$ & $\circ$ & $\circ$ & & & & & $v_{55}+v_{57} A$ \\
\hline $1090 v w$ & $\circ$ & o & $1095 \mathrm{vw}$ & $1091 w$ & 0 & 4 & $1090 \mathrm{vw}$ & 1091 & o & $\circ$ & $v_{17}, a, v_{52} b, \rho \mathrm{CH}_{3}(86), \delta \mathrm{CH}_{3}(13)$ \\
\hline $1039 \mathrm{~s}$ & 1 & 5 & $1049 \mathrm{~m}$ & $1041 w$ & $\circ$ & 4 & 1050w & 1048 & 1 & 6 & $v_{18} \mathrm{a}, v_{52} \mathrm{~b}, \mathrm{PCH}_{3}(57), v \mathrm{CH}_{3}-\mathrm{N}(39)$ \\
\hline 977 vs & 3 & 4 & $9858, \mathrm{sh}$ & $977 \mathrm{ve}$ & 3 & 2 & & & & & $v_{55}+v_{59} A$ \\
\hline $970 v s$ & 31 & 3 & $976 \mathrm{vs}$ & $977 \mathrm{vs}$ & 30 & 2 & $986:(0.23)$ & 982 & 22 & 3 & 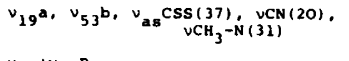 \\
\hline \multirow[t]{2}{*}{$952 \mathrm{~s}$} & -2 & 4 & $960 \mathrm{~s}, \mathrm{sh}$ & $958 \mathrm{~m}, \mathrm{sh}$ & 0 & 7 & $964 \mathrm{w}, \mathrm{sh}$ & & & & $v_{26}+v_{55} B$ \\
\hline & & & & $898 \mathrm{vw}$ & 0 & 0 & & & & & $2 \cdot v_{2} 4^{A}$ \\
\hline $849 \mathrm{~s}$ & $-2 / 13$ & 2 & $855 \mathrm{~m}$ & 852: & $0 / 13$ & 2 & $8578(0.32)$ & $\left\{\begin{array}{l}857 \\
856\end{array}\right.$ & 7 & $\begin{array}{l}3 \\
3\end{array}$ & $\begin{array}{l}v_{2} 2 a^{a}, \mathrm{VCH}_{3}-\mathrm{N}(81) \\
v_{54} \mathrm{vCH}_{3}-\mathrm{N}(82)\end{array}$ \\
\hline \multirow[t]{2}{*}{$839 w$, sh } & 2 & 2 & & & & & & & & & $v_{24}+v_{25} A$ \\
\hline & & & & $824 v w$ & 3 & & & & & & $v_{22}+v_{27} A$ \\
\hline \multirow[t]{2}{*}{$790 v w$} & 1 & 1 & & $795 \mathrm{vw}$ & 2 & 2 & & & & & $2 \cdot v_{58} A$ \\
\hline & & & & $773 \mathrm{vw}$ & $\circ$ & $\circ$ & & & & & $2 \cdot v_{25^{A}}$ \\
\hline $674 \mathrm{vw}$ & & & & $676 \mathrm{vw}$ & 2 & 0 & & 676 & 3 & 4 & $v_{21} \mathrm{a}, \delta\left(\mathrm{CH}_{3}\right)^{\prime} \mathrm{N}(5 \mathrm{O}), \mathrm{OCH}_{3}(13)$ \\
\hline $667 \mathrm{vw}$ & & & & $667 \mathrm{vw}$ & & & & 670 & 3 & 5 & $v_{55} \mathrm{~b}, \delta\left(\mathrm{CH}_{3}\right) 2_{2} \mathrm{~N}(57), \mathrm{DCH}_{3}(14)$ \\
\hline
\end{tabular}


Table 2. Continued.

\begin{tabular}{|c|c|c|c|c|c|c|c|c|c|c|c|}
\hline \multicolumn{4}{|c|}{ Infrared ${ }^{a}$} & \multicolumn{4}{|c|}{ Raman $^{a}$} & & & & \multirow{3}{*}{$\begin{array}{l}\text { Assignment and Description }{ }^{C} \\
(\text { PED, } 8)^{d}\end{array}$} \\
\hline \multicolumn{3}{|c|}{ solid, disc } & \multirow{2}{*}{$\begin{array}{l}\text { solution } \\
\mathrm{CHCl}_{3}\end{array}$} & \multicolumn{2}{|c|}{ solid } & \multirow[b]{2}{*}{$15_{\mathrm{N}-\mathrm{sh}}$} & \multirow{2}{*}{$\begin{array}{l}\text { solution } \\
\mathrm{CHCl}_{3}\end{array}$} & \multicolumn{3}{|c|}{ Calculated ${ }^{b}$} & \\
\hline parent & ${ }^{13} \mathrm{c}-\mathrm{sh}$ & ${ }^{15} \mathrm{~N}-\mathrm{sh}$ & & parent & ${ }^{13} \mathrm{c}-\mathrm{sh}$ & & & parent & ${ }^{13} \mathrm{c}-\mathrm{an}$ & 15 & \\
\hline $563 \mathrm{~m} / \mathrm{w}$ & 3 & 3 & $563 \mathrm{~m} / \mathrm{w}$ & $561 \mathrm{vs}$ & 2 & 3 & $565 \mathrm{ve}(0.14)$ & 558 & $\circ$ & $\circ$ & $v_{22^{a}}{ }^{a}, v s-s(75)$ \\
\hline $542 \mathrm{w}, \mathrm{sh}$ & $16 / 10$ & 3 & $540 \mathrm{w}$ & $536 \mathrm{wm}, \mathrm{en}$ & & 3 & $542 \mathrm{vw}, \mathrm{sh}$ & 538 & 13 & 4 & $v_{23^{a}} a t \operatorname{tCN}(65), t C-S(22)$ \\
\hline \multirow[t]{4}{*}{$520 v w$, sh } & 12 & $\circ$ & & & & & & 526 & 12 & 5 & $v_{56} b, \operatorname{tcN}(69), \operatorname{tc}-s(22)$ \\
\hline & & & & $502 \mathrm{vw}$ & 1 & 0 & $502 \mathrm{vw}$ & & & & $2 \cdot v_{27^{A}}$ \\
\hline & & & & 490vw & $\circ$ & $\circ$ & $493 \mathrm{vm}$ & & & & $v_{57}+v_{66} A$ \\
\hline & & & $475 \mathrm{vm}$ & $480 v w$ & 0 & $\circ$ & & & & & $v_{59}+v_{61} A$ \\
\hline $441 \mathrm{~m}$ & $\circ$ & 1 & $444 \mathrm{~m}$ & 443* & 0 & 1 & $4478(0.28)$ & $\left\{\begin{array}{l}442 \\
39\end{array}\right.$ & $\circ$ & 2 & $\begin{array}{l}v_{5} 7^{\mathrm{b}}, \quad \mathrm{vC}=\mathrm{S}(36), \delta \mathrm{CH} \mathrm{HC}_{3} \mathrm{NC}(40) \\
v_{24} 4^{\mathrm{a}}, \mathrm{vC}=\mathrm{S}(38), \delta \mathrm{CH}_{3} \mathrm{NC}(39)\end{array}$ \\
\hline $396 \mathrm{~m}$ & 0 & 1 & $396 \mathrm{~m}$ & 395. & $\circ$ & 1 & $3958(0.20)$ & $\left\{\begin{array}{l}395 \\
390\end{array}\right.$ & 0 & 0 & 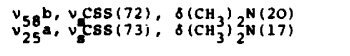 \\
\hline $358 w$ & 0 & $\circ$ & & $361 \%$ & $\circ$ & $\circ$ & & & & & $2 \cdot v_{61} \wedge$ \\
\hline $312 \mathrm{vw}, \mathrm{sh}$ & 0 & 0 & & $317 \mathrm{~m}$ & 1 & 1 & $314 \mathrm{~m}(0.5)$ & 312 & $\circ$ & 1 & $v_{59} b, \delta C-s-s(24), \delta S=C-s(33)$ \\
\hline $284 w, s h$ & $\circ$ & 2 & & 2836 & & & & 290 & 1 & 4 & $v_{26} a, \operatorname{tCN}(54), \operatorname{tC}-\mathrm{s}(16)$ \\
\hline $275 m$ & 1 & 5 & & $267 \mathrm{~m}$ & $\circ$ & $\circ$ & & 266 & 2 & 3 & $v_{60} b, \operatorname{tcN}(66), \operatorname{tc}-s(15)$ \\
\hline 268v, sh & & & & $255 \mathrm{wn}$ & & & & 252 & 1 & 1 & $v_{27^{a}}, \underset{\operatorname{deN}(12)}{\delta S-C-S(39, \delta N C S(16)}$ \\
\hline \multirow[t]{2}{*}{$218 \mathrm{vw}, \mathrm{br}$} & & & & & & & & & & & $v_{33}+v_{61} B$ \\
\hline & & & & $203 n$ & $\circ$ & 0 & & & & & $2 \cdot v_{32^{A}}$ \\
\hline $178 m$ & & & & $179 m$ & 0 & 1 & $175 \mathrm{~m}(0.6)$ & 175 & 0 & $\circ$ & $v_{61} \mathrm{~b}, 8 \mathrm{NCS}(64), 8 \mathrm{CH}_{3} \mathrm{NC}(17)$ \\
\hline $151 \mathrm{vw}$ & & & & $158 \mathrm{vw}$ & & & & & & & $v_{32}+v_{66} B$ \\
\hline $144 \mathrm{vw}$ & & & & $162 \mathrm{vw}$ & & & & $e^{145}$ & o & $\circ$ & $v_{28^{a}}, \mathrm{TCH}_{3}(95)$ \\
\hline \multirow[t]{2}{*}{$135 \mathrm{vw}$} & & & & $133 \mathrm{vw}$ & 0 & 0 & & $\left\{\begin{array}{l}136 \\
133 \\
133 \\
131\end{array}\right.$ & $\begin{array}{l}1 \\
: \\
: \\
0\end{array}$ & $\begin{array}{l}\circ \\
: \\
: \\
\circ\end{array}$ & 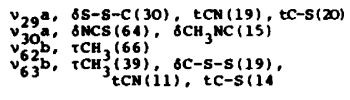 \\
\hline & & & & $125 \mathrm{vw}$ & 1 & 0 & & $\left\{\begin{array}{l}125 \\
124\end{array}\right.$ & $\stackrel{\circ}{\circ}$ & $\stackrel{\circ}{\circ}$ & $\begin{array}{l}v_{31} \mathrm{a}, \quad \mathrm{TCH}_{3}(87) \\
\mathrm{v}_{64} \mathrm{~b}, \mathrm{TCH}_{3}(80)\end{array}$ \\
\hline $117 \%$ & & & & 116w & $\circ$ & 1 & & & & & lattice mode \\
\hline \multirow[t]{2}{*}{$110 w$} & & & & $110 m$ & $\circ$ & 0 & & & & & lattice mode \\
\hline & & & & $97 \mathrm{vu}$ & & & & 96 & $\circ$ & $\circ$ & $v_{32} a, \operatorname{tc}-s(55), \operatorname{tcN}(34)$ \\
\hline $91 w$, sh & & & & & & & & 93 & 0 & o & $v_{65} b, \operatorname{tc}-s(65), \operatorname{tCN}(29)$ \\
\hline $84 \mathrm{~m}$ & & & & 75. & 2 & & & & & & lattice mode \\
\hline $68 \mathrm{w}$ & & & & $64 m, \mathrm{eh}$ & $\circ$ & $\circ$ & & & & & Lattice mode \\
\hline \multirow[t]{2}{*}{ sow } & & & & & & & & 51 & $\circ$ & $\circ$ & $v_{66} b, t c-s(61), \delta c-s-s(25)$ \\
\hline & & & & 44: & $\circ$ & & & 47 & $\circ$ & $\circ$ & $v_{33^{a}}, \operatorname{tc}-s(60), 6 C-s-s(21)$ \\
\hline $38 \mathrm{vw}$ & & & & $37 m$, sh & $\circ$ & & & 39 & $\circ$ & $\circ$ & $v_{34^{a}}, t s-s(65)$ \\
\hline $32 \mathrm{w}$ & & & & & & & & & & & lattice mode \\
\hline
\end{tabular}

a The following abbreviations have been used: a, strong, m, medium; $w$, weak, br, broad; sh, shoulder. The Raman depolarisation ratio is given in parenthesis. Weak bands between 1550 and $2750 \mathrm{~cm}^{-1}$ have been omitted.

biteration based upon all isotopic species.

Abbreviations: $v=$ stretching, $\delta, \Delta=$ deformation, $p=$ rocking, $w=$ wagging, $t=$ twisting, $\tau=$ torsion, and, as subscripts,

$s=$ symmetric, as = antisymetric.

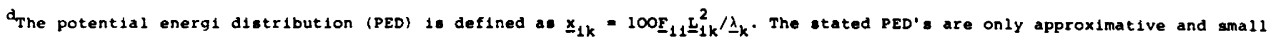
contributions have been neglected.

mental $v_{19} a$ at $970 \mathrm{~cm}^{-1}$ by $31 \mathrm{~cm}^{-1}$ to $939 \mathrm{~cm}^{-1}$, while the two other bands are considered combination modes which are hardly displaced on ${ }^{13} \mathrm{C}$ substitution. Though this interpretation is supported by the Raman spectra the results remain ambiguous.

Raman depolarisation measurements have been performed for both TMTD and TMTD- $d_{12}$ in
$\mathrm{CHCl}_{3}$ solution. The fundamentals belonging to species $a$ should give rise to polarised Raman lines whereas those of species $b$ should appear depolarised. However, the in-phase and out-of-phase combinations in most cases superimpose to give one band which appears polarised. Therefore, only the assignments of $v_{23} a, v_{59} b$, and $v_{61} b$ in the 
spectrum of TMTD and $v_{21} a, v_{22} a, v_{59} b$, and $v_{61} b$ in TMTD- $d_{12}$ are supported from these measurements.

The $\mathrm{CH}_{3}$ and $\mathrm{CD}_{3}$ stretching modes. The four asymmetric $\mathrm{CH}$ stretching modes of species $a$ are expected to fall within a narrow frequency range $\left(7 \mathrm{~cm}^{-1}\right.$ according to the NCA) coincident with the corresponding modes of species $b$. Furthermore, the NCA predicts the symmetric modes to coincide to one band at a lower frequency. The two strongest bands found both in the IR and the Raman spectrum of TMTD have been assigned to these overlapping fundamentals (Table 2). Some of the remaining bands are easily explained as overtones or binary

Table 3. Observed and calculated vibrational frequencies $\left(\mathrm{cm}^{-1}\right)$ of perdeuterotetramethylthiouram disulfide and frequency shifts obtained by comparison with $\left[\left(\mathrm{CD}_{3}\right)_{2} \mathrm{~N}-{ }^{13} \mathrm{CSS}\right]_{2}$.

\begin{tabular}{|c|c|c|c|c|c|c|c|c|}
\hline \multicolumn{3}{|c|}{ Infrared ${ }^{a}$} & \multicolumn{3}{|c|}{$\operatorname{Raman}^{a}$} & & & \multirow{3}{*}{$\begin{array}{l}\text { Assignment and Description }{ }^{c} \\
{\text { (PED, },)^{d}}^{\text {d }}\end{array}$} \\
\hline soldd, dis & & \multirow{2}{*}{$\begin{array}{l}\text { solution } \\
\mathrm{CHCl}_{3}\end{array}$} & \multicolumn{2}{|c|}{ sol1d } & \multirow{2}{*}{$\begin{array}{c}\text { solution } \\
\mathrm{CHCl}_{3}\end{array}$} & \multicolumn{2}{|c|}{ Calculated ${ }^{b}$} & \\
\hline parent & ${ }^{13} \mathrm{c}-\mathrm{sh}$ & & parent & ${ }^{13} \mathrm{c}-\mathrm{mh}$ & & parent & ${ }^{13} \mathrm{c}-\mathrm{sh}$ & \\
\hline \multirow[t]{2}{*}{$2262 w, b r$} & & $2265 \mathrm{w}, \mathrm{sh}$ & $2265 \mathrm{w}, \mathrm{br}$ & & $2270 \mathrm{w}, \mathrm{eh}$ & & & $v_{8}+v_{11} A$ \\
\hline & & $2240 \mathrm{~m}, \mathrm{sh}$ & $2232 \mathrm{~m}, \mathrm{sh}$ & & $2240 \mathrm{~m}, \mathrm{sh}$ & 2220 & $\circ$ & $v_{1} a, v_{35} b, v_{a s} C D(95)$ \\
\hline $2221 w$ & $\circ$ & $2233 \mathrm{~m}$ & 22238 & 2 & $2232=(0.50)$ & $\left\{\begin{array}{l}2204 \\
2200 \\
2199\end{array}\right.$ & $\begin{array}{l}\circ \\
\circ\end{array}$ & 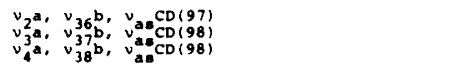 \\
\hline $2176 w$ & 3 & $2190 \mathrm{w}$ & $2177 w$ & 4 & $2188 \mathrm{~W}$ & & & $v_{9}+v_{11} A$ \\
\hline $2135 w$ & 3 & $2143 \mathrm{~m}$ & $2135 \mathrm{~s}$ & 4 & $2243=(0.23)$ & & & $v_{10}+v_{1} 1^{A}$ \\
\hline \multirow[t]{2}{*}{$2108 \mathrm{w}$} & $\circ$ & $2114 w, 8 h$ & $2113 \mathrm{w}, \mathrm{sh}$ & & $2115 w$, sh & & & $2 \cdot v_{11}^{A}$ \\
\hline & & $2107 \mathrm{~m}$ & $2102 m$ & 2 & $2105 m(0.33)$ & & & $2 \cdot v_{12^{A}}$ \\
\hline $2087 \mathrm{vw}$ & $\circ$ & $2094 w, s h$ & $2090 \mathrm{w}, \mathrm{sh}$ & & $2093 \mathrm{w}, \mathrm{sh}$ & & & $2 \cdot v_{13^{A}}$ \\
\hline $2060 \mathrm{~m}$ & $\circ$ & 20708 & $2062 \mathrm{~m}$ & 1 & $2070 \mathrm{~s}(0.28)$ & $\left\{\begin{array}{l}2066 \\
2065\end{array}\right.$ & $\stackrel{\circ}{\circ}$ & 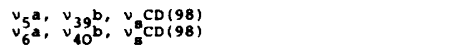 \\
\hline $1538 \mathrm{vw}$ & 8 & & $1540 \mathrm{vw}$ & & & & & $2 \cdot v_{20^{A}}$ \\
\hline $1505 \mathrm{vw}, \mathrm{sh}$ & & & $1509 \mathrm{vw}$ & & 21500vw, br & & & $v_{16}+v_{21} A$ \\
\hline $1443 \mathrm{vs}, \mathrm{sh}$ & 2 & & & & $1447 \mathrm{~m}, \mathrm{sh}$ & & & $v_{12}+v_{24} A$ \\
\hline $1437 \mathrm{~s}$ & 22 & $1437 \mathrm{vs}$ & 14338 & 21 & $1438 \mathrm{~s}(0.79)$ & 1434 & 26 & $v_{7} a, v_{41} b, v C N(70)$ \\
\hline $1419 \mathrm{w}, \mathrm{sh}$ & & & $1417 \mathrm{vw}, \mathrm{sh}$ & & $1418 \mathrm{vw}, \mathrm{sh}$ & & & $v_{51}+v_{56} A$ \\
\hline $1296 \mathrm{vw}$ & 2 & & $1302 \mathrm{vw}$ & & & & & $v_{20}+v_{22} A$ \\
\hline $1258 \mathrm{vw}, \mathrm{sh}$ & & & $1259 \mathrm{vw}$ & & & & & $v_{15}+v_{27^{A}}$ \\
\hline $1210 \mathrm{~s}$ & 4 & & $1214 w$ & 4 & & 1211 & 8 & $v_{8} a, v_{42} b, v C D_{3}-N(64), \delta C D_{3} \mathrm{NC}(42)$ \\
\hline $1166 \mathrm{vw}$ & 2 & & $1156 \mathrm{vw}$ & & & & & $2 \cdot v_{21} A$ \\
\hline \multirow[t]{2}{*}{$1147 \mathrm{vw}$} & 7 & & $1144 v w$ & 5 & & & & $v_{20}+v_{25}$ \\
\hline & & & $1124 \mathrm{vw}, \mathrm{sh}$ & & & & , & $2 \cdot V_{55} \mathrm{~A}$ \\
\hline $1111 m$ & 3 & $1120 \mathrm{~m}$ & $1112 \mathrm{w}$ & & $1119 w(0.9)$ & $\begin{array}{l}1111 \\
1106\end{array}$ & 1 & $\begin{array}{l}v_{9} a, v_{4} b, \delta C D_{3}(84), v C D_{3}-N(24) \\
v v_{3} a, v_{3} b, \delta C D_{3}(80), v C D_{3}-N(32)\end{array}$ \\
\hline \multirow[t]{2}{*}{$1100 \mathrm{w}, \mathrm{sh}$} & 0 & $1104 w$, th & $\begin{array}{l}1104 w, s h \\
1078 w\end{array}$ & $\begin{array}{l}3 \\
1\end{array}$ & & 1106 & & $\begin{array}{l}v_{10^{a}, v_{14} b^{b}, \delta D_{3}(80)}\left(v^{\prime} D_{3}-N(32)\right. \\
v_{18^{+v_{2}}} 7^{A}\end{array}$ \\
\hline & & & $1074 w, s h$ & 1 & & & & $v_{54}+v_{59} A$ \\
\hline $1057 \mathrm{~s}$ & 2 & & $1058 w$ & 2 & $21064 \mathrm{w}, \mathrm{br}$ & 1053 & $\circ$ & $v_{11} a, v_{45} b, 8 C_{3}(87)$ \\
\hline $1042 \mathrm{vw}, \mathrm{sh}$ & & & $1045 \mathrm{ww}$ & & & $\left\{\begin{array}{l}1050 \\
1045 \\
1043\end{array}\right.$ & $\begin{array}{l}\circ \\
:\end{array}$ & 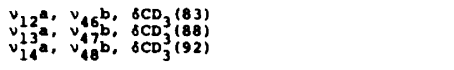 \\
\hline 10178 & $16 / 4$ & $1012 \mathrm{~m}$ & $1013 \mathrm{~m}$ & 15 & $1021 \mathrm{~m}(0.35)$ & 1006 & 11 & $v_{15} a, v_{49} b, D^{2} D_{3}(37), \delta\left(C D_{3}\right)_{2}^{N(17), v C s(12)}$ \\
\hline $946 w, s h$ & $\circ$ & & $942 w$ & & 944w & & & $v_{21}+v_{25} A$ \\
\hline $925 \mathrm{vw}, \mathrm{sh}$ & & & $928 \mathrm{w}, \mathrm{eh}$ & $\circ$ & $928 w$, en & 928 & $\circ$ & $v_{16^{a}}, v_{50^{b}}, Q \mathrm{CD}_{3}(75)$ \\
\hline 9108 & $0 / 8$ & 9148 & 914. & 8 & $9218(0.60)$ & 927 & 18 & $v_{1}, a, v_{51} b, v_{a s} \operatorname{css}(29), v C N(18), v C D_{3}-N(17)$ \\
\hline $889 \mathrm{~m}$ & 12 & 8998 & $892 \mathrm{~m}$ & 10 & $899 m, \operatorname{sh}(0.81)$ & & & $v_{22}+v_{25} A$ \\
\hline $837 \mathrm{mu}$ & $\circ$ & & B34w & $\circ$ & $833 w, e h$ & 832 & $\circ$ & $v_{18^{a}}, v_{52} b, \rho C_{3}(92)$ \\
\hline $816 \mathrm{~m}$ & 2 & & $817 w$ & 1 & $824 w(0.7)$ & 824 & 1 & $v_{1} 9^{a}, v_{5} 3^{b}, 8 C_{3}(80)$ \\
\hline $770 \mathrm{~m}$ & 6 & & $773 m$ & 5 & & $\left\{\begin{array}{l}770 \\
769\end{array}\right.$ & $\begin{array}{l}3 \\
3\end{array}$ & 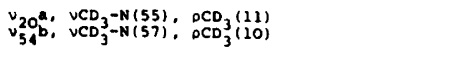 \\
\hline $750 \mathrm{w}$ & 2 & & $753 \mathrm{vw}$ & & & & & $2 \cdot v_{25^{A}}$ \\
\hline $618 \mathrm{vi}$ & & & $615 \mathrm{w}$ & & & & & $v_{25}+v_{27} A$ \\
\hline $572 w$ & 1 & $576 \mathrm{w}$ & $\begin{array}{l}585 \mathrm{vw} \\
573 \mathrm{~m}\end{array}$ & 1 & $578 \mathrm{~m}(0.45)$ & 589 & ○ & $\begin{array}{l}2 \cdot v_{59^{A}} \\
v_{22} a^{a}, v S-S(43), P C D_{3}(12), 8\left(C_{3}\right)_{2}^{N}(12)\end{array}$ \\
\hline
\end{tabular}

Acta Chem. Scand. A 32 (1978) No. 8 
Table 3. Continued.

\begin{tabular}{|c|c|c|c|c|c|c|c|c|}
\hline \multicolumn{3}{|c|}{ Infrared ${ }^{a}$} & \multicolumn{3}{|c|}{$\operatorname{Raman}^{a}$} & & & \multirow{3}{*}{ 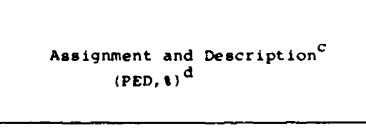 } \\
\hline \multicolumn{2}{|c|}{ solid, disc } & \multirow{2}{*}{$\begin{array}{c}\text { solution } \\
\mathrm{CHCl}_{3}\end{array}$} & \multicolumn{2}{|c|}{ sol1d } & \multirow{2}{*}{$\begin{array}{c}\text { solution } \\
\mathrm{CHCl}_{3}\end{array}$} & \multicolumn{2}{|c|}{ Calculated ${ }^{b}$} & \\
\hline parent & ${ }^{13} \mathrm{c}-\mathrm{sh}$ & & parent & ${ }^{13} \mathrm{c}-\mathrm{sh}$ & & parent & ${ }^{13} \mathrm{c}-\mathrm{sh}$ & \\
\hline $566 \mathrm{w}, \mathrm{sh}$ & & & $566 \mathrm{w}, \mathrm{sh}$ & & $567 w, s h$ & 559 & 1 & $v_{55} \mathrm{~b}, \delta\left(\mathrm{CD}_{3}\right) 2_{2} \mathrm{~N}(33), \mathrm{OCD}_{3}(27)$ \\
\hline $539 \mathrm{w}, \mathrm{sh}$ & & $538 \mathrm{w}, \mathrm{sh}$ & $544 \mathrm{w}, \mathrm{sh}$ & & & & & $2 \cdot v_{26} \mathrm{~A}$ \\
\hline $528 \mathrm{vs}$ & 2 & $532 \mathrm{~s}$ & $529 \mathrm{~s}$ & 1 & $528 \mathrm{v}=(0.31)$ & 528 & 1 & $v_{22} \mathrm{a}, v s-s(37), \delta\left(\mathrm{CD}_{3}\right) 2_{2}^{\mathrm{N}(28)}$ \\
\hline $518 \mathrm{vw}$ & $6 / 10$ & & $522 w, 8 h$ & & & 520 & 13 & $v_{23^{a}}, \operatorname{tcN}(58), \operatorname{tc}-5(22)$ \\
\hline $509 \mathrm{vw}$ & 19 & $504 \mathrm{vw}, \mathrm{sh}$ & $505 \mathrm{vw}, \mathrm{sh}$ & & $504 \mathrm{vw}, \mathrm{sh}$ & 506 & 13 & $v_{56} b, \operatorname{tCN}(64), \operatorname{tc}-s(24)$ \\
\hline $487 \mathrm{vw}, \mathrm{br}$ & $\circ$ & $480 \mathrm{vw}, \mathrm{sh}$ & $484 \mathrm{vw}, \mathrm{sh}$ & & & & & $2 \cdot v_{27} \mathrm{~A}$ \\
\hline \multirow[t]{2}{*}{$396 \mathrm{~m}$} & 2 & & $400 \mathrm{~m}$ & 2 & $400 \mathrm{~m}$ & $\left\{\begin{array}{l}410 \\
406\end{array}\right.$ & $\stackrel{\circ}{\circ}-1$ & 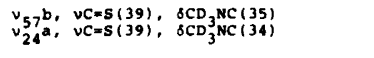 \\
\hline & & & $387 \mathrm{w}$ & & $390 \mathrm{~m}$ & & & $v_{26}+v_{62} B$ \\
\hline $375 m$ & 1 & & $375 s$ & $\circ$ & & $\left\{\begin{array}{l}379 \\
377\end{array}\right.$ & $\stackrel{\circ}{\circ}$ & 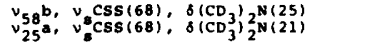 \\
\hline $342 v w$ & o & & $343 m$ & $\circ$ & $340 \mathrm{w}$ & & & $2 \cdot v_{61} B$ \\
\hline $298 \mathrm{~m}$ & 2 & & $300 \mathrm{~s}$ & o & $2938(0.6)$ & 301 & $\circ$ & $v_{59} b, \delta c-s-s(21), \delta s=c-s(34)$ \\
\hline \multirow[t]{2}{*}{$2: 1 \mathrm{~m}$} & $\circ$ & & $269 w$ & $\circ$ & & 271 & 2 & $v_{26} a, \operatorname{tCN}(48), \operatorname{tC}-\operatorname{s}(17)$ \\
\hline & & & $255 \mathrm{vw}$ & & & 249 & 1 & $v_{60^{b}}, \operatorname{tcN}(65), \operatorname{tC}-s(17)$ \\
\hline $246 \mathrm{~m}$ & & & $242 \mathrm{~m}$ & $\circ$ & & 242 & $\circ$ & $v_{27^{a}}, \delta S=C-S(39), \quad \delta N C S(15), V C N(12)$ \\
\hline $207 \mathrm{vw}$ & & & & & & & & $v_{30^{+v}} v_{63^{B}}$ \\
\hline $193 \mathrm{vw}$ & & & $192 \mathrm{vw}$ & $\circ$ & & & & $v_{63}+v_{64} A$ \\
\hline $178 w$ & & & $177 \mathrm{~m}$ & $\circ$ & $171 \mathrm{~m}(0.5)$ & 168 & $\circ$ & $v_{61} b, \delta N C S(59), \delta C D_{3} N C(18)$ \\
\hline $158 w$ & & & $160 \mathrm{w}$ & & $160 \mathrm{w}(0.7)$ & & & $v_{34}+v_{62} B$ \\
\hline $138 \mathrm{vw}$ & & & $132 \mathrm{vw}$ & 0 & & 132 & 1 & $v_{28} a, \delta S-s-C(39), \operatorname{tcc}(17), \operatorname{tC}-s(21)$ \\
\hline $122 \mathrm{vw}$ & & & $125 \mathrm{ww}$ & $\circ$ & & $\left\{\begin{array}{l}127 \\
123\end{array}\right.$ & $\stackrel{1}{0}$ & 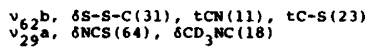 \\
\hline \multirow[t]{2}{*}{$117 \mathrm{~m}$} & & & $115 w$ & o & $115 w$ & 112 & $\circ$ & $v_{30} \mathrm{a}, \tau \mathrm{CD}_{3}(93)$ \\
\hline & & & $105 m$ & o & $202 w$ & 98 & $\circ$ & $v_{63} \mathrm{~b}, \operatorname{TCD}_{3}(66), t C-s(22), \operatorname{tCN}(12)$ \\
\hline $89 \mathrm{w}, \mathrm{sh}$ & & & $93 w$ & $\circ$ & & $\left\{\begin{array}{l}90 \\
89\end{array}\right.$ & : & $v_{64}^{v_{31}} a_{b}^{a}, \operatorname{TCD}_{3} \operatorname{TCD}_{3}(74), t C-s(14)$ \\
\hline $83 w$ & & & & & & $\left\{\begin{array}{l}87 \\
82\end{array}\right.$ & : & $\begin{array}{l}v_{32} \mathrm{a}, \operatorname{TCD}_{3}(25), \operatorname{tcN}(30), \operatorname{tC}-s(41) \\
v_{65} \mathrm{~b}, \operatorname{TCD}_{3}(33), \operatorname{tCN}(21), \operatorname{tC}-\mathrm{s}(44)\end{array}$ \\
\hline $76 \mathrm{vw}$ & & & $76 \mathrm{vw}$ & & & & & lattice mode \\
\hline $67 w$ & & & $67 \mathrm{~s}$ & o & & & & lattice mode \\
\hline $61 \mathrm{~m}$ & & & $63 \mathrm{~m}, \mathrm{sh}$ & $\circ$ & & & & lattice mode \\
\hline $56 m$ & & & & & & & & lattice mode \\
\hline \multirow[t]{2}{*}{$43 w$} & & & $43 \mathrm{~s}$ & 0 & & $\left\{\begin{array}{l}47 \\
14\end{array}\right.$ & $\stackrel{\circ}{\circ}$ & 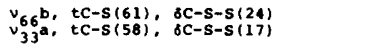 \\
\hline & & & $37 m, s h$ & $\circ$ & & 38 & $\circ$ & $v_{34^{a}}, i s-S(62)$ \\
\hline
\end{tabular}

asee footnote to Table 2 for abbreviations. Weak absorption in the range $1550-200 \mathrm{~cm}^{-1}$ has been omitted.

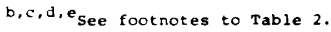

combination modes. However, the ${ }^{13} \mathrm{C}$ and ${ }^{15} \mathrm{~N}$ shifts of three weak bands in the upper part of this region $\left(2960-3017 \mathrm{~cm}^{-1}\right)$ are negligible and must stem from combination modes of fundamentals which also remain unchanged. Since no such fundamentals are available they probably arise through ternary combination modes.

In the spectra of TMTD- $d_{12}$ (Table 3) the pattern is quite similar, except that one of the asymmetric CD stretching bands $\left(v_{1} a, v_{35} b\right)$ is predicted to be shifted $c a .20 \mathrm{~cm}^{-1}$ towards higher frequency relative to the other asymmetric $\mathrm{CD}$ stretching vibrations. These fundamentals have been assigned to the strong rather broad absorption near 2230 $\mathrm{cm}^{-1}$ with a prominent shoulder at $c a .2240 \mathrm{~cm}^{-1}$. The NCA predicts the four symmetric CD stretching modes to be coincident at $c a .2065 \mathrm{~cm}^{-1}$ corresponding to the strongest band in the IR spectrum in this region. However, in the Raman spectrum of TMTD- $d_{12}$ in the solid phase the strongest band in this region occurs at $2135 \mathrm{~cm}^{-1}$. In $\mathrm{CHCl}_{3}$ solution this band moves to $2143 \mathrm{~cm}^{-1}$ and becomes comparable in intensity to the band at $2070 \mathrm{~cm}^{-1}$. Since the NCA strongly contradicts this to be a

Acta Chem. Scand. A 32 (1978) No. 8 
fundamental it is instead assumed to be an unusually strong combination mode. Only a combination of the $\mathrm{CD}_{3}$ deformation modes $\left(e . g . v_{10}+v_{14} A\right)$ can be expected to achieve the necessary intensity enhancement by Fermi resonance with the fundamentals of this region, and the band has been assigned accordingly. The assignment of the remaining bands of TMTD- $d_{12}$ is straightforward.

The thioureide band $v_{7} / v_{41}$. In the IR spectrum of TMTD, the prominent band near $1500 \mathrm{~cm}^{-1}$ (known as the thioureide band ${ }^{15}$ ) is assigned to the overlapping fundamentals $v_{7} a$ and $v_{41} b$. Coleman's results showed ${ }^{3}$ this band to consist essentially of CN stretching $(81 \%)$ weakly coupled to $\mathrm{C}=\mathrm{S}$ and $\mathrm{CH}_{3}-\mathrm{N}$ stretching. The results of the present NCA (including full treatment of the $\mathrm{CH}_{3}$ groups instead of considering them to be point masses) rather indicate this band to consist of CN stretching $(29 \%)$ strongly coupled to $\mathrm{CH}_{3}$ deformation and rocking (total $60 \%$ ). On deuteration the coupling is removed and the corresponding band in TMTD- $d_{12}$ near $1435 \mathrm{~cm}^{-1}$ consists mainly of CN stretching $\left(70{ }^{\circ}{ }_{0}\right)$. Though our NCA probably overestimates the contribution of $\mathrm{CH}_{3}$ modes to this band in TMTD (the ${ }^{13} \mathrm{C}$ and ${ }^{15} \mathrm{~N}$ shifts are calculated too small) there is hardly any doubt that both $\mathrm{CN}$ stretching and $\mathrm{CH}_{3}$ deformation and rocking modes contribute to this band. On complex formation with cobalt(II) chloride the frequency of this vibration is raised by $30 \mathrm{~cm}^{-1}$ reflecting the concomitant increase in double bond character of the $\mathrm{CN}$ bond. ${ }^{5}$

The $\mathrm{CH}_{3}$ and $\mathrm{CD}_{3}$ deformation and rocking vibrations. The four $\delta_{\mathrm{as}} \mathrm{CH}_{3}$ and two $\delta_{\mathrm{s}} \mathrm{CH}_{3}$ modes of species $a$ are predicted by the NCA to be coincident with the corresponding six bands of species $b$ and to appear in the range $1380-1470 \mathrm{~cm}^{-1}$ in the spectrum of TMTD. Since only four bands are observed in this region, the broad and unresolved absorption occurring in both the Raman and IR spectrum of TMTD near $1450-1460 \mathrm{~cm}^{-1}$ has been assigned to the overlapping fundamentals $v_{8}-v_{10} a / v_{42}-v_{44} b$. In the case of $v_{13} a / v_{47} b$ the observation of ${ }^{13} \mathrm{C}\left(8 \mathrm{~cm}^{-1}\right)$ and ${ }^{15} \mathrm{~N}\left(9 \mathrm{~cm}^{-1}\right)$ shifts supports the occurrence of a weak coupling to $\mathrm{CN}$ stretching as indicated by the calculations. In the spectrum of TMTD- $d_{12}$ (Table 3 ) the corresponding $\mathrm{CD}_{3}$ deformation modes occur in the range $1040-1120 \mathrm{~cm}^{-1}$ and are easily assigned in accordance with the results of the NCA.

The $\rho \mathrm{CH}_{3}$ modes are expected from the calculations to give rise to four bands in the $1000-1200$ $\mathrm{cm}^{-1}$ region each consisting of overlapping fundamentals of species $a$ and $b$. On ${ }^{13} \mathrm{C}$ substitution, part of the strong band near $1150 \mathrm{~cm}^{-1}$ (Table 2) is displaced by $13-15 \mathrm{~cm}^{-1}$ towards lower frequencies while part of the absorption remains unchanged. The NCA indicates that the fundamentals $v_{15} a / v_{49} b$ are distinguished by a major ${ }^{13} \mathrm{C}$ shift of $12 \mathrm{~cm}^{-1}$ arising from coupling with the $\mathrm{CN}$ stretching mode while the $\rho \mathrm{CH}_{3}$ mode $v_{16} a / v_{50} b$ occurs at almost identical frequencies but is not diplaced by ${ }^{13} \mathrm{C}$ substitution. The $1150 \mathrm{~cm}^{-1}$ band is therefore assigned to all four fundamentals. The remaining fundamentals are assigned following the condition that $v_{17} a / v_{51} b$ is neither displaced by ${ }^{13} \mathrm{C}$ nor by ${ }^{15} \mathrm{~N}$ substitution, while $v_{18} a / v_{52} b$ should shift by ca. $6 \mathrm{~cm}^{-1}$ on ${ }^{15} \mathrm{~N}$ substitution as a result of coupling to the $\mathrm{CH}_{3}-\mathrm{N}$ stretching mode. A few remaining weak bands in this region are assigned to overtones and combination modes.

Three of the $\rho \mathrm{CD}_{3}$ modes in TMTD- $d_{12}$ should give rise to bands which are not displaced by ${ }^{13} \mathrm{C}$ substitution and, again, with complete overlap between fundamentals of species $a$ and $b$. These have been identified with the weak absorption occurring near 928,833 and $824 \mathrm{~cm}^{-1}$. The overlapping fundamentals $v_{15} a / v_{49} b$, however, are calculated to exhibit a ${ }^{13} \mathrm{C}$-shift of $11 \mathrm{~cm}^{-1}$ because of coupling with the $\mathrm{C}=\mathrm{S}$ stretching and $\left(\mathrm{CD}_{3}\right)_{2} \mathrm{~N}$ deformation modes. These are assigned the medium to strong bands observed in both the IR and Raman spectra near $1015 \mathrm{~cm}^{-1}$ displaying a ${ }^{13} \mathrm{C}$ shift of $15-16 \mathrm{~cm}^{-1}$.

The skeletal modes of TMTD in the range $800-$ $1300 \mathrm{~cm}^{-1}$. In the IR spectrum of TMTD a strong band occurs at $1245 \mathrm{~cm}^{-1}\left(\mathrm{CHCl}_{3}\right)$ which is shifted $4 \mathrm{~cm}^{-1}$ on ${ }^{13} \mathrm{C}$ substitution and $19 \mathrm{~cm}^{-1}$ on ${ }^{15} \mathrm{~N}$ substitution towards lower frequencies. The Raman counterpart is much weaker but occurs at identical frequencies and with almost identical isotopic shifts. In good agreement with the NCA this band is assigned as overlapping $v_{14} a / v_{48} b$ fundamentals due to $\mathrm{CH}_{3}-\mathrm{N}$ stretching $(51 \%)$ weakly coupled to $\mathrm{CH}_{3} \mathrm{NC}$ deformation and $\mathrm{CH}_{3}$ rock. In TMTD- $d_{12}$ the coupling with $\mathrm{CH}_{3}$ rock is removed and the corresponding band $v_{8} a / v_{42} b$ is found at $c a .30$ $\mathrm{cm}^{-1}$ lower frequencies. Coleman et al. ${ }^{3}$ assigned this band to $\mathrm{CH}_{3}$ rocking, but this seems excluded by the isotopic shifts reported here.

One of the strongest bands in both the IR and Raman spectrum of TMTD is observed near 980 $\mathrm{cm}^{-1}$. The ${ }^{15} \mathrm{~N}$ shift is only ca. $2 \mathrm{~cm}^{-1}$ but on ${ }^{13} \mathrm{C}$ substitution it displays a large shift of $30 \mathrm{~cm}^{-1}$. 
These characteristics allow an unambiguous assignment to the overlapping fundamentals $v_{19} a / v_{53} b$ arising from an out-of-phase stretching mode of the $\left(\mathrm{CH}_{3}\right)_{2} \mathrm{~N}-\mathrm{CSS}$ group of TMTD involving $v_{\text {as }} \mathrm{CSS}-$ $(37 \%), v \mathrm{CN}(20 \%)$ and $v \mathrm{CH}_{3}-\mathrm{N}(31 \%)$. In TMTD$d_{12}$ the counterpart is calculated to appear at 917 $\mathrm{cm}^{-1}$ displaying a ${ }^{13} \mathrm{C}$ shift of $18 \mathrm{~cm}^{-1}$. However, two strong bands occur near this position in both the IR and Raman spectra of TMTD- $d_{12}$, one near $915 \mathrm{~cm}^{-1}\left({ }^{13} \mathrm{C}\right.$-shift $\left.8 \mathrm{~cm}^{-1}\right)$ and one near $890 \mathrm{~cm}^{-1}$ $\left({ }^{13} \mathrm{C}\right.$ shift $\left.10-12 \mathrm{~cm}^{-1}\right)$. Since the sum of the observed ${ }^{13} \mathrm{C}$ shifts corresponds quite closely to the predicted value, an explanation could be the occurrence of an overtone or combination mode with the following features: (i) The ${ }^{13} \mathrm{C}$ shift should be negligible since the observed ${ }^{13} \mathrm{C}$ shifts of this region can be explained entirely as arising from the fundamental. (ii) At least one of the components should involve skeletal modes of the $\left(\mathrm{CH}_{3}\right)_{2} \mathrm{~N}-\mathrm{CSS}$ group in order that Fermi resonance with the $v_{17} a / v_{51} b$ fundamental may achieve the necessary intensity. In our opinion the assigned combination mode, $v_{22}+v_{25} A$, most closely corresponds to these demands.

The overlapping fundamentals $v_{20} a / v_{51} b$ are calculated to appear at $c a .855 \mathrm{~cm}^{-1}$ with small ${ }^{15} \mathrm{~N}$ and ${ }^{13} \mathrm{C}$ shifts. Considering the PED in comparison with the elements of the $L$-matrix, it consists of more than $80 \%$ of symmetric $\left(\mathrm{CH}_{3}\right)_{2} \mathrm{~N}$ stretching. Except for some ambiguity in the interpretation of the experimental ${ }^{13} \mathrm{C}$ shifts these features agree with the experimental spectra. In TMTD- $d_{12}$ weak coupling occurs to the $\mathrm{CD}_{3}$ rocking mode,

The region below $700 \mathrm{~cm}^{-1}$ of TMTU and $T M T U-d_{12}$. The band observed at $565 \mathrm{~cm}^{-1}$ in the Raman spectrum of TMTD in $\mathrm{CHCl}_{3}$ solution is the strongest band in the spectrum; furthermore, it is the band having the lowest depolarisation ratio (0.14). It must accordingly be of species $a$ and originate in vibrations producing a strong Raman scattering. The counterpart in the IR spectrum is only of weak to medium strength indicating the change of dipole moment during the vibration to be small. The observed ${ }^{13} \mathrm{C}$ and ${ }^{15} \mathrm{~N}$ shifts are negligible $\left(2-3 \mathrm{~cm}^{-1}\right)$. These features indicate strongly (cf. Bellamy ${ }^{16}$ ) that this band should be assigned to $v_{22} a$, due to the $\mathrm{S}-\mathrm{S}$ stretching mode $(75 \%$ according to our NCA). The corresponding force constant $K_{\mathrm{L}}=2.58 \mathrm{mdyn} / \AA$, is intermediate between the values for aliphatic sulfides $(3.10$ mdyn $/ \AA^{17}$ ) and the value proposed by Coleman et al. for $\operatorname{TMTD}^{3}(2.30 \mathrm{mdyn} / \AA)$. The latter value is obtained on the assumption, that the band with maximum $\mathrm{S}-\mathrm{S}$ stretching character $(91 \%$ according to their NCA) is instead the strong, polarised Raman band at $447 \mathrm{~cm}^{-1}$ also observed with weak to medium strength in the IR at $444 \mathrm{~cm}^{-1}$. Their NCA indicate the band at $565 \mathrm{~cm}^{-1}$ to originate in a vibration involving stretching and bending of the $\mathrm{CH}_{3}-\mathrm{N}-\mathrm{C}-\mathrm{S}$ part of the molecule, which, in our opinion, is not likely to be an adequate description of the strongest band in the Raman spectrum. Unfortunately, the results obtained for TMTD- $d_{12}$ do not unambigously support our interpretation, since the $\mathrm{S}-\mathrm{S}$ stretching character is equiparted between the two strong Raman bands at 578 and $528 \mathrm{~cm}^{-1}\left(\mathrm{CHCl}_{3}\right)$, the latter of which also appear very strong in the IR contrary to expectations. If Coleman's assignment was correct, the band originating primarily in $\mathrm{S}-\mathrm{S}$ stretching in the spectrum of TMTD- $d_{12}$ should be the Raman line of medium strength at $400 \mathrm{~cm}^{-1}$ also having an IR counterpart of similar intensity. At present a decisive interpretation cannot be given for these bands.

Torsional vibrations of the $\mathrm{C}_{2} \mathrm{~N}-\mathrm{C}-\mathrm{S}-\mathrm{S}-\mathrm{NC}_{2}$ chain give rise to the fundamentals $v_{23}, v_{26}, v_{32}, v_{33}$, and $v_{34} a$ and $v_{56}, v_{60}, v_{65}$, and $v_{66} b$. These are only tentatively identified except in the case of $v_{23} a$ and $v_{56} b$, which are distinguished by displaying ${ }^{13} \mathrm{C}$ shifts calculated to $c a .13 \mathrm{~cm}^{-1}$. In TMTU they appear as two weak bands at $c a$. 540 and $520 \mathrm{~cm}^{-1}$ and in TMTU- $d_{12}$ they are displaced by $c a .20$ $\mathrm{cm}^{-1}$ towards lower frequencies. In both cases the ${ }^{13} \mathrm{C}$ shifts have been difficult to establish with certainty, especially in the Raman spectrum where this region is completely dominated by the strong $v_{22} a$. Though the shifts are somewhat irregular, varying from 6 to $19 \mathrm{~cm}^{-1}$ according to the IR spectra, we believe these fundamentals to be correctly identified. They were not observed by Coleman et al. ${ }^{3}$, likewise vibrations concerned with out-of-plane deformations and torsions were not included in their NCA. According to our NCA, some coupling occur to the $\tau \mathrm{CH}_{3}$ bands of TMTD in the $120-50 \mathrm{~cm}^{-1}$ range and to the $\tau \mathrm{CD}_{3}$ bands of TMTU- $d_{12}$ between 80 and $120 \mathrm{~cm}^{-1}$.

The symmetric stretching vibration of the CSS group is expected ${ }^{2}$ to contribute significantly to strong Raman bands occurring in the 400-500 $\mathrm{cm}^{-1}$ region. In the Raman spectrum of TMTU we observe two strong, polarised bands at 447 and 395 $\mathrm{cm}^{-1}\left(\mathrm{CHCl}_{3}\right)$ which might both comply with these demands. The NCA indicates the band at highest frequencies to originate in $\mathrm{C}=\mathrm{S}$ stretching (36- 
$38 \%$ ) coupled to $\mathrm{CH}_{3} \mathrm{NC}$ deformation $(39-40 \%$ ), of which the former component explains the observed Raman intensity. The Raman line near $395 \mathrm{~cm}^{-1}$ is predominantly due to the $v_{\mathrm{s}} \mathrm{CSS}$ mode $\left(72-73 \%\right.$ ) but with a weak coupling to $\left(\mathrm{CH}_{3}\right)_{2} \mathrm{~N}$ deformation $(17-20 \%)$. This assignment is in agreement with the shifts observed on deuteration. It differs considerably from the assignments of this region by Coleman et al. however, their agreement between observed and calculated values in this range is not good.

The two skeletal deformation modes, $v_{59} b$ due to the $\mathrm{S}=\mathrm{C}-\mathrm{S}-\mathrm{S}$ chain and $v_{27} a$ originating in the $\mathrm{N}-\mathrm{CS}_{2}$ group, are assigned to bands near 315 and $255 \mathrm{~cm}^{-1}$, respectively, in the spectra of TMTD. In the spectra of TMTD- $d_{12}$ both bands are shifted towards lower frequencies and are found near 300 and $245 \mathrm{~cm}^{-1}$, respectively. These assignments are based exclusively upon the agreement with the results of the NCA. The in-phase and out-of-phase combinations of NCS deformation (with small contributions from $\mathrm{CH}_{3} \mathrm{NC}$ deformation $v_{30} a$ and $v_{61} b$, are assigned to bands near 135 and $175 \mathrm{~cm}^{-1}$ in TMTD. The latter band has been observed in $\mathrm{CHCl}_{3}$ solution as a Raman line with depolarisation ratio 0.6 , not far from full depolarisation $(0.75)$. The counterparts in the spectrum of TMTD- $d_{12}$ are quite similar. The two bands originating from NCS deformation were calculated by Coleman et al. ${ }^{3}$ to fall near 79 and $92 \mathrm{~cm}^{-1}$, which is at much lower frequencies than our assignment for TMTD. However, their force constants for $\mathrm{S}=\mathrm{C}-\mathrm{S}$ and $\mathrm{S}=\mathrm{C}-\mathrm{N}$ bending were found to be 1.98 and 0.13 mdyn $\AA /(\mathrm{rad})^{2}$, respectively, which seems to be a much more unrealistic result than the values found by us (Table 1). Furthermore, their calculated values for the region below $600 \mathrm{~cm}^{-1}$ seem to be severely hampered by the omission of torsional vibrations which, as discussed above, give rise to several bands in this region.

Acknowledgements. We thank Mrs. J. E. Gustavsen who recorded many of the IR and Raman spectra and Dr. Kjartan Marøy, Bergen for making some unpublished results concerning the structure of TMTD available to us. This research was supported by grants from the Danish Natural Science Research Council and the Norwegian Research Council for Science and the Humanities.

\section{REFERENCES}

1. Anthoni, U., Borch, G., Gustavsen, J., Klæboe, P. and Nielsen, P. H. Spectrochim. Acta A 33 (1977) 403.

2. Dahl, B. M., Jensen, K. A., Nielsen, P. H. and Borch, G. Acta Chem. Scand. 25 (1971) 2029, 2039; 26 (1972) 2241.

3. Coleman, M. M., Koenig, J. L. and Shelton, J. R. J. Phys. Sci., Polym. Phys. Ed. 12 (1974) 1001.

4. Gore, R. C., Hannah, R. W., Pattacini, S. C. and Porro, T. J. J. Assoc. Off. Anal. Chem. 54 (1971) 1040.

5. Contreras, H. and Cortés, H. Inorg. Nucl. Chem. Lett. 6 (1970) 225.

6. Brinkhoff, H. C., Grotens, A. M. and Steggerda, J. J. Recl. Trav. Chim. Pays-Bas 89 (1970) 11; Marøy, K. Personal communications.

7. Brinkhoff, H. C. and Grotens, A. M. Recl. Trav. Chim. Pays-Bas 111 (1971) 252.

8. Wilson, N. K. J. Phys. Chem. 75 (1971) 1067.

9. Schlottmann, B. U. Tetrahedron Lett. (1971) 4051.

10. Edgar, B. L., Duffy, D. J., Palazzotto, M. C. and Pignolet, L. H. J. Am. Chem. Soc. 95 (1973) 1125.

11. Fraser, R. R., Boussard, G., Saunders, J. K., Lambert, J. B. and Mixan, C. E. J. Am. Chem. Soc. 93 (1971) 3822.

12. Wilson, E. B., Jr. J. Chem. Phys. 7 (1939) 1047; 9 (1941) 76.

13. Snyder, R. G. and Schachtschneider, J. H. Spectrochim. Acta 21 (1965) 169.

14. Knoeck, J. and Witt, J. Spectrochim. Acta A 32 (1976) 149.

15. Jensen, K. A. and Nielsen, P. H. Acta Chem. Scand. 20 (1966) 597.

16. Bellamy, L. J. The Infra-red Spectra of Complex Molecules, 3rd Ed., Chapman \& Hall, London 1975.

17. Scott, D. W. and El-Sabban, M. Z. J. Mol. Spectrosc. 30 (1969) 317; 31 (1969) 362.

Received May 8, 1978. 